\title{
Three dimensional confocal microscopy of fluorescent microspheres: imaging and size determination
}

\author{
Y.P. Rakovich ${ }^{* a}$, L. Yang ${ }^{\text {b }}$, C.M. Taylor ${ }^{\text {a }}$, L.A. Dunbar ${ }^{\text {c }}$, A. Mac Raighne ${ }^{\text {a }}$ J.F. Donegan ${ }^{\text {a }}$, \\ E. M. McCabe ${ }^{\text {a }}$ \\ ${ }^{a}$ Semiconductor Photonics Group, Department of Physics, Trinity College, Dublin 2, Ireland \\ ${ }^{\mathrm{b}}$ Institute for Experimental Physics, Freie Univerisity Berlin, Germany \\ ${ }^{\mathrm{c}}$ Institut de Photonique et d'Electronique Quantique, EPFL, CH-1015 Lausanne, Switzerland
}

\begin{abstract}
In this paper, we experimentally studied both the bright-field and fluorescence images of microspheres by conventional and confocal scanning polarization microscopes. A qualitative analysis have been given to show a physical picture on the imaging of the microspheres. Emission spectra from melamine formaldehyde microspheres stained with Ethidium Bromide or covered by thin shell of CdTe nanocrystals have been experimentally studied. We adopted analytical expressions describing the resonance spacing in order to determine the size of the microspheres.
\end{abstract}

Key words: Three-dimensional spectroscopy, scattering particles, whispering gallery modes.

\section{INTRODUCTION}

The inelastic light-scattering and emission properties of spherical microcavities are presently attracting considerable attention in quantum optics, laser physics, material science, and device technology ${ }^{1}$. For dielectric microsphere of high quality factor $Q$ and radius $a$, which is large compared with the wavelength of light $(\lambda)$, extremely narrow resonant structure can be observed in emission or scattering spectra ${ }^{2}$. Such a whispering gallery mode (WGM) oscillations result from multiple total internal reflection of light waves at the spheresurrounding media interface and occur at specific values of the size parameter $x=2 \pi a / \lambda$. The distribution of emission intensity within the microsphere is shown to be very sensitive to excitation geometry when the incident light wave is resonant with a WGM ${ }^{3-5}$. The existence of resonant WGM is reported for microdroplets, spherical microresonators made from polymer or glass spheres doped with dye molecules or rare-earth ions ${ }^{2}$, however only recent years one can see a dramatic progress in development of new microstrustures: spherical microcavities doped with nanoscopic light emitters - semiconductor nanocrystals (NCs) ${ }^{6-7}$. Coupling NCs (quantum dots) to a high-Q WGM of a microsphere can provide a strong coherent interaction between the WGM and the electronic states of the dots. Due to the effect of quantum confinement in quantum dots, this new concept allows one to tune both the modes of microcavities and NCs into resonance providing the most efficient mode coupling ${ }^{8}$.

In this paper, we experimentally studied both the bright-field and fluorescence images of microspheres by conventional and confocal scanning polarization microscopes. A qualitative analysis have been done to show a physical picture on the imaging of the microspheres. Emission spectra from melamine formaldehyde microspheres stained with Ethidium Bromide or covered by thin shell of CdTe nanocrystals have been experimentally studied and analyzed. We presented an approach for WGM identification and size determination of a spherical microcavity.

\section{EXPERIMENTAL DETAILS}

Figure 1 shows the experimental setup. An $\mathrm{HeNe}(632.8 \mathrm{~nm})$ or $\mathrm{Ar}^{+}(457.9 \mathrm{~nm})$ laser is focused onto the microsphere samples through an objective lens with a high numerical aperture (NA) of 0.9 . The laser is initially polarized in the vertical direction, which we define as the $\mathrm{x}$ direction. In micro-PL experiments only the emission light above $515 \mathrm{~nm}$ from the sample can pass through the filter cube. By flipping the mirror M4, the light can be input into either a scanning fluorescence microscope with a photomultiplier tube or a spectrometer with a cooled CCD camera. In backscatter imaging experiment, the filter cube was replaced by a beam splitter and the light from the sample was focused onto a $30 \mu \mathrm{m}$ diameter pinhole by a tube lens (L3). The computer

*Yury.Rakovich@tcd.ie; phone +353-1-608-2193; fax 353-1-671-1759 
used to control a motorized stage moving in the $\mathrm{x}$ and $\mathrm{y}$ directions and a piezoelectric actuator driving the objective lens in $\mathrm{z}$ direction. When the pinhole is removed, considering the size of the detector and objective lens as infinitely large, the sectioning property of the system will vanish and the system works as a conventional scanning microscope.

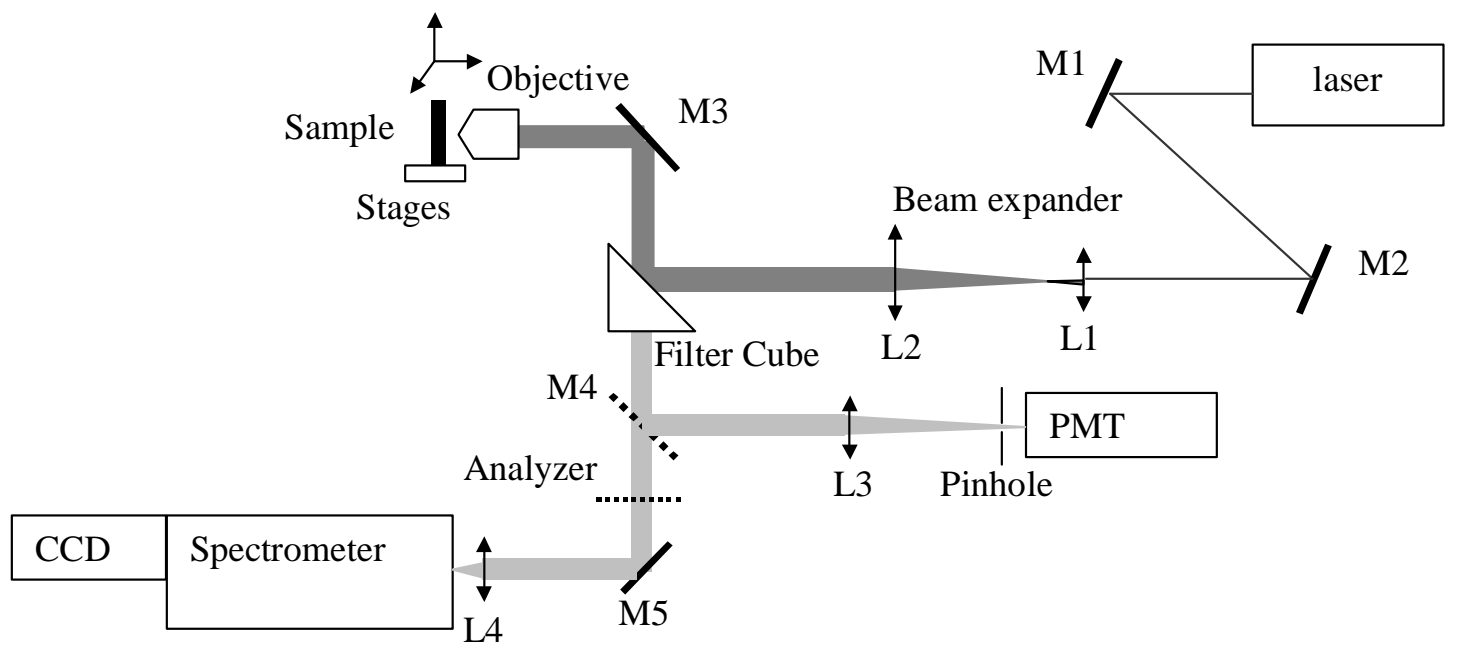

Figure: 1. Experimental setup.

The microsphere samples, melamine formaldehyde (MF) microspheres (Microparticles GmbH, Germany) stained with Ethidium Bromide (EtBr) dye or covered by thin shell of CdTe nanocrystals ${ }^{9,}{ }^{10}$, have diameters from 2 to $5 \mu \mathrm{m}$.

\section{RESULTS AND DISCUSSION}

\subsection{Imaging by Conventional Polarization Microscopy}

First we experimentally studied backscattered images by using the conventional scanning microscope where the pinhole shown in fig. 1 was removed. First we focused on the center plane of the spheres. Fig. 2(a)-(c) are the images with parallel, crossed analyzer and no analyzer, respectively. Interference rings can still be seen within the region of the spheres in fig. 2(a) and (c).
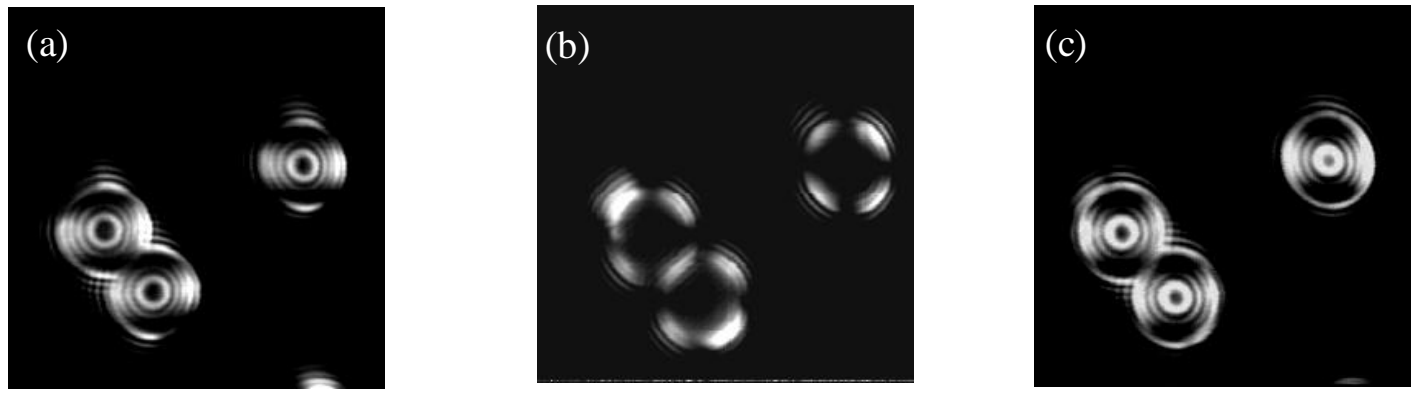

Figure 2: Normalized images of the microspheres by conventional scanning optical microscope with parallel analyzer (a), crossed analyzer (b) and no analyzer (c), at $\mathrm{z}=0$.

It is well known that for the conventional scanning microscope, the detected signal $\mathrm{I}_{\text {conv }}$ scattered from the edge of the sphere is the integral of the intensity over the exit pupil of the objective lens and can be calculated as ${ }^{11}$

$$
I_{\text {conv }}=\int_{0}^{2 \pi} \int_{0}^{\alpha}|E|^{2} \sin \theta \cos \theta d \theta d \phi .
$$

where the field $\mathbf{E}=\mathrm{E}_{\mathrm{x}} \mathbf{i}+\mathrm{E}_{\mathrm{y}} \mathbf{j}, \theta$ is the backscattering angle, $\phi$ is the polar angle measured from the normal $\mathbf{n}$.

The $\mathrm{x}$ and $\mathrm{y}$ component of the field in the exit pupil of the objective can be expressed as 


$$
\begin{aligned}
& \mathrm{E}_{\mathrm{x}}=\mathrm{P}(\beta(\theta, \varphi)+\mathrm{Q}(\beta(\theta, \varphi) \cos 2(\varphi+\xi), \\
& \mathrm{E}_{\mathrm{y}}=\mathrm{Q}(\beta(\theta, \varphi) \sin 2(\varphi+\xi)
\end{aligned}
$$

where $\mathrm{P}$ and $\mathrm{Q}$ are the backscattering amplitudes $\mathrm{P}(\beta, \theta, \phi)=\mathrm{S}_{/ /}(\beta, \theta, \phi)+\mathrm{S}_{\perp}(\beta, \theta, \phi)$ and $\mathrm{Q}(\beta, \theta, \phi)=\mathrm{S}_{/ /}(\beta, \theta, \phi)$ $S_{\perp}(\beta, \theta, \phi)$ and $\beta$ is the size parameter, defined as $\left.\beta=2 \pi \mathrm{a} / \lambda\right)$.

We consider the scattering amplitudes to be symmetrical as $S_{/ /}(\phi)=S_{/ /}(2 \pi-\phi)$ and $S_{\perp}(\phi)=S_{\perp}(2 \pi-\phi)$. When the analyzer is parallel to the incident polarization, from Eq. 1 and Eq. 2, the detected intensity can be expressed by

$$
\mathrm{I}_{/ /}^{\mathrm{conv}}=\int_{0}^{2 \pi \alpha}\left[\mathrm{P}^{2}+2 \mathrm{PQ} \cos 2 \phi \cos 2 \xi+\mathrm{Q}^{2} \cos ^{2} 2 \phi \cos ^{2} 2 \xi\right] \sin \theta \cos \theta \mathrm{d} \theta \mathrm{d} \phi .
$$

Because the intensity is mainly modulated by the factor $\cos ^{2} 2 \xi$, it reaches a maximum at the angle $\xi=0$, $\pi / 2, \pi$, and $3 \pi / 2$ and minimum at $\xi=\pi / 4,3 \pi / 4,5 \pi / 4$ and $7 \pi / 4$ along edge of the sphere. Due to the effect of the second integral term, the intensity at $\xi=0$ or $\pi$ are slightly different from that at $\xi=\pi / 2$ or $3 \pi / 2$, as shown in fig. 2(a).

Substituting Eq. 3 into Eq. 1, the distribution of the intensity for a crossed analyzer can be obtained as:

$$
\mathrm{I}_{\perp}^{\text {conv }}=\left(\sin ^{2} 2 \xi\right) \cdot \int_{0}^{2 \pi \alpha} \int_{0}^{2} \mathrm{Q}^{2} \cos ^{2} 2 \phi \sin \theta \cos \theta \mathrm{d} \theta \mathrm{d} \phi,
$$

where the multiplying factor $\sin ^{2} 2 \xi$ predicts that four arcs will be located at the angle of $45^{\circ}$ and a dark cross will appear in the image, as shown in fig. 2(b).

Finally, when the analyzer is removed, the signal from the sphere can be expressed as

$$
\mathrm{I}_{\text {conv }}=\int_{0}^{2 \pi \alpha} \int_{0}^{2}\left[\mathrm{P}^{2}+2 \mathrm{PQ} \cos 2 \phi \cos 2 \xi+\mathrm{Q}^{2} \cos ^{2} 2 \phi\right] \sin \theta \cos \theta \mathrm{d} \theta \mathrm{d} \phi .
$$

The image of the sphere edge slightly varies with different $\xi$, which is modulated by the second integral term of $\mathrm{PQ}(\beta, \theta, \phi) \cos 2 \phi$ over $\phi$ and the factor $\cos 2 \xi$, as shown in fig. $2(\mathrm{c})$.

\subsection{The three-dimensional bright-field imaging of microspheres in confocal microscope}

In order to control the optical quality of the samples we utilize the sectioning property of the confocal scanning microscope. The images are shown in fig. 3. An isolated sphere and a pair of touching spheres are observed. The light was first focused on the surface of the substrate $(z=-a)$ (near the back surface of the sphere) and only the shadow of the sphere can be seen in fig. 3(a). When the focal plane is moved toward the equatorial plane of the sphere $(\mathrm{z}=0)$, bright rings appear in the periphery of the sphere fig. $3(\mathrm{~b})$.
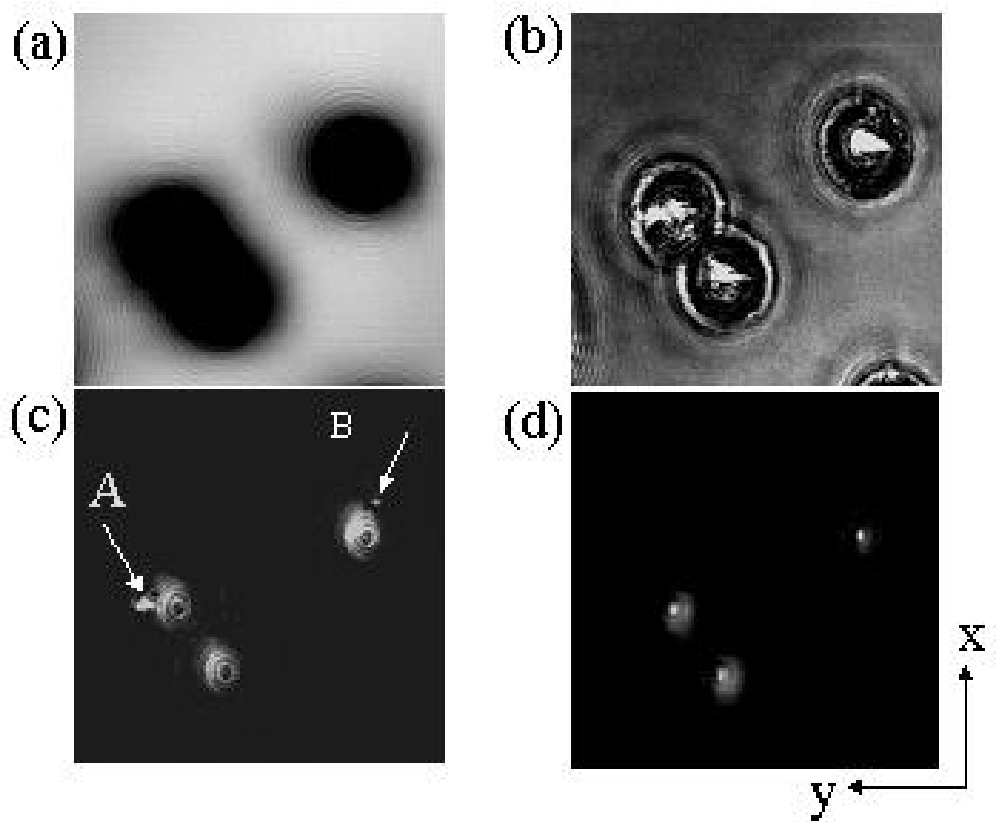

Figure 3: Normalized confocal scanning images of microsphere, $5.2 \mu \mathrm{m}$ in diameter, with different axial locations. From (a)-(d): $\mathrm{z}=-\mathrm{a}, 0, \mathrm{a} / 2$, and $\mathrm{a}$, respectively. Two defects $\mathrm{A}$ and $\mathrm{B}$ are inside the sphere. 
Since the distance from the plane $\mathrm{z}=0$ to the substrate is about $2.6 \mu \mathrm{m}$ and the FWHM of the system axial response of $\sim 0.8 \mu \mathrm{m}$ is considerably less than this, light from the off-focused substrate will be blocked by the pinhole. In addition, other light scattering away from the focus will be also blocked by the pinhole. Hence bright rings can only be due to the light backscattered from the focused point of the sphere surface.

The bright center in fig. 3(b) can be explained in terms of geometrical optics: when the light is focused on the center of the sphere, rays propagate along radial directions and reflect back. In order to see the rings around the sphere surface, we increased the gain of the detector, and as a result, the center of the sphere is saturated in fig. 3(b). Fig. 3 (c)-(d) shows the images near $\mathrm{z}=\mathrm{a} / 2$ and a, respectively, with the same gain of the detector which is comparatively lower than that in fig. 2(b). Two point-like defects A and B can be clearly seen inside the sphere near the region between $z=a / 2$, which cannot be observed by conventional microscopy due to the poor sectioning property.

\subsection{Emission images of microspheres}

Fig. 4(a) shows the emission image of a single microsphere stained with EtBr with homogeneous PL distribution. Similar distribution can be observed for $\mathrm{CdTe} / \mathrm{MF}$ system because in that case microspheres are covered by only very thin shell (1-3 monolayers) of nanocrystals. In contrast, Fig. 4(b) shows the images of another EtBr sphere with a bright spot at the edge and a tail opposite. The images have been investigated spectrally to make sure that the signal from the laser light is lower than -20db. We attribute the feedback loop pattern in Fig. 4(b) to the electromagnetic field distribution integrated over entire emissions outside a microsphere with unevenly stained dye. We notice that there are no obvious interference rings inside the sphere in the emission image compared with the bright field image shown in Fig. 2(c) where the reflected laser light is detected. This is due to the incoherent property of fluorescence light. In addition, we observed that the emission image is unaffected by the angular position of an analyzer which indicates that dye molecules are in random orientation and position.
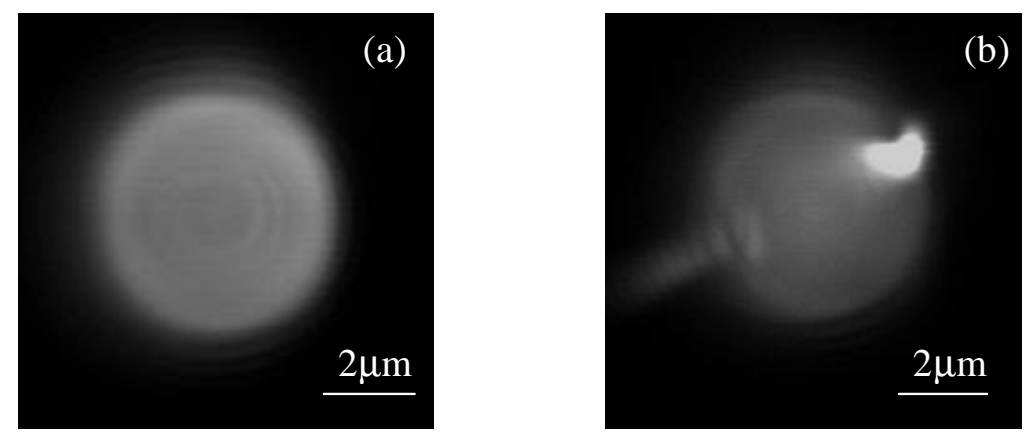

Figure 4: Emission images of two different microspheres stained with EtBr dye.

Combining emission confocal imaging technique with three-dimensional bright-field analysis of microspheres we can therefore control the quality of the spherical microcavity, the spatial emission distribution, locate any defects, and then select the spheres of highest quality for spectroscopic measurements.

\subsection{Spectral properties of emitting microspheres and size determination}

In contrast to the broad, featureless PL band in the spectra of dye solution or colloidal NCs, the emission spectra of a single microsphere stained with EtBr or covered by thin shell of CdTe nanocrystals exhibit very sharp periodic structure (Fig. 5). The observed peak structure is a result of coupling of photon states of microsphere and electronic states of dye molecules or NCs. As one can see from Figure 5, the WGM peaks which are superimposed on a background signal arising from part of dye or NCs emission, which does not match any WGM of the microsphere.

The placement and spacing between WGM peaks is determined by the size and refractive index. In the absence of gain, the placement of the WGM resonances can be characterized by a mode number (angular quantum number) $\mathrm{n}$, which is equal to the circumference divided by the wavelength of the light propagating within the microsphere.

As one can see from Figure 5, the WGM peaks with different $\mathrm{n}$ are superimposed on a background signal arising from part of NCs emission, which does not match any WGM of the microsphere. The placement and spacing between WGM peaks is determined by the size and refractive index of the microsphere while spectral intensity distribution depends on the parameters of NCs and can be easily modified by using NCs of different size. 

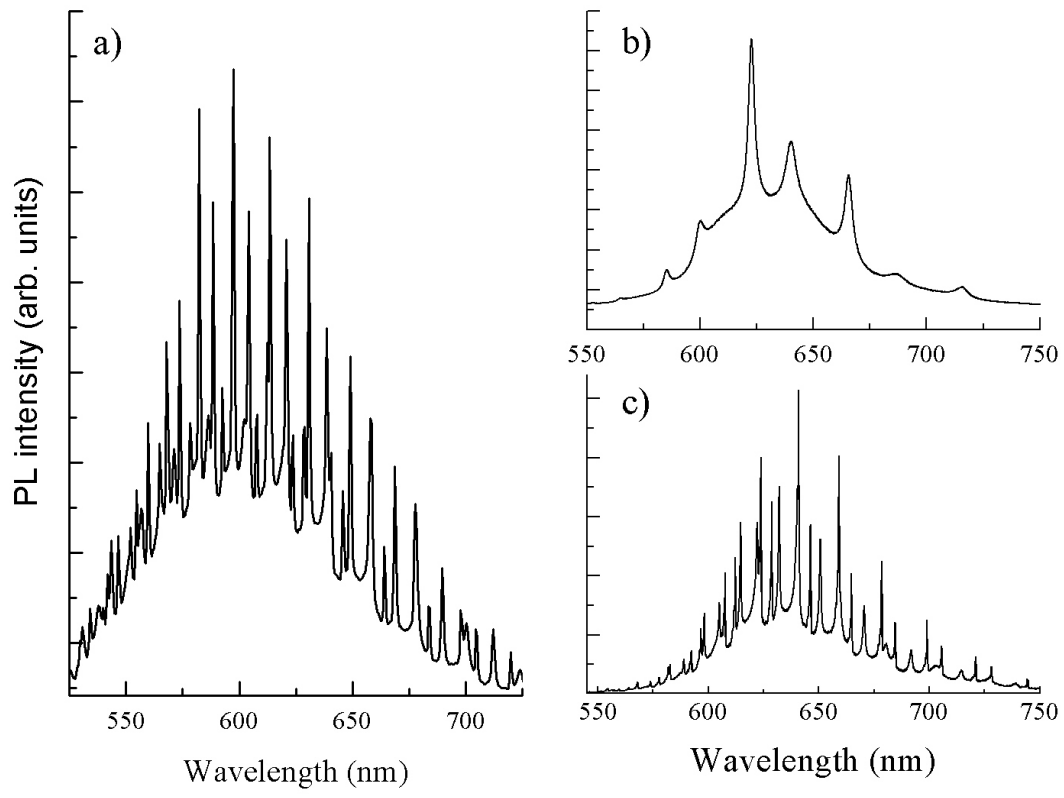

Figure 5: PL spectrum from a single microsphere stained with EtBr dye (a) and covered by 1 monolayer of CdTe NCs (b,c). Microsphere size is about $5.2 \mu \mathrm{m}(\mathrm{a}, \mathrm{c})$ and $2.2 \mu \mathrm{m}(\mathrm{b})$.

For the WGM peaks shown in Fig. 5 the value of the angular quantum number $n$ is much higher than the size parameter $x=\pi d / \lambda$ throughout the whole spectral region ( $\mathrm{d}$ is the diameter of the microsphere). In this case the approximate distance between two resonances of successive modes with the same order and polarization can be obtained from the expression ${ }^{12}$ :

$$
\Delta \lambda=\frac{\lambda_{\mathrm{n}}{ }^{2} \tan ^{-1}\left\{\mathrm{~m}^{2}-1\right\}^{1 / 2}}{\pi \mathrm{d}\left\{\mathrm{m}^{2}-1\right\}^{1 / 2}},
$$

where $\lambda_{n}$ is the emission wavelength of the WGM peaks with different $n$, and $m$ is refractive index of MF sphere, i.e. 1.68 .

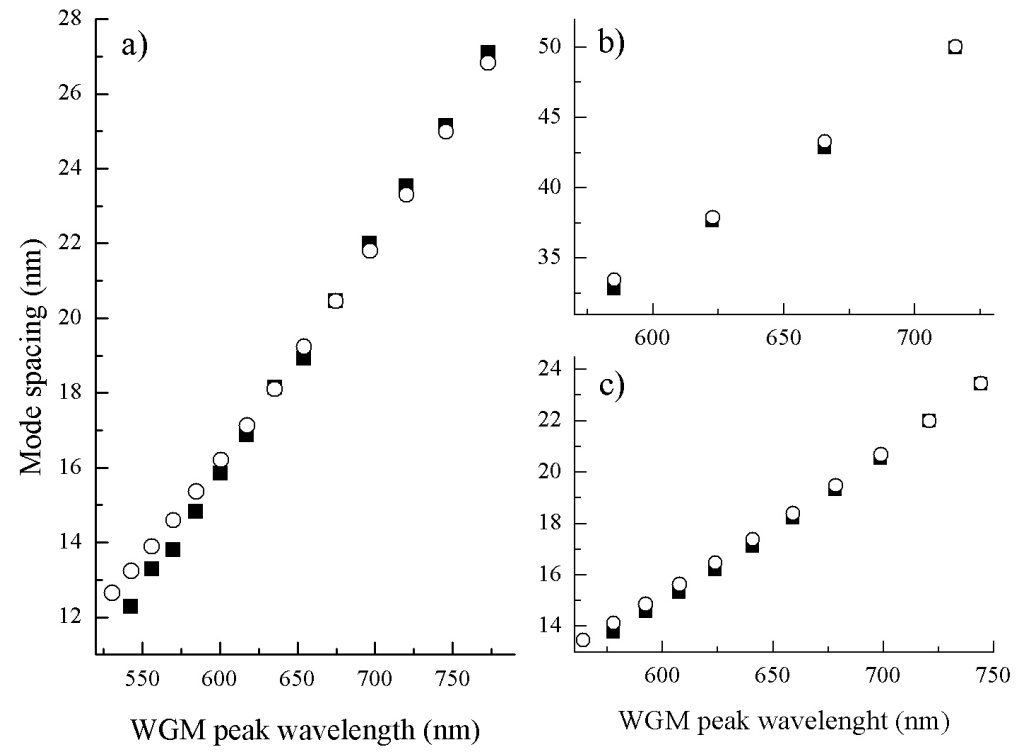

Figure 6: Experimental (squares) and calculated (circles) spacing between adjacent WGM for microsphere stained with EtBr dye (a) and covered by 1 monolayer of CdTe NCs (b,c). Estimated microsphere size is $5.11 \mu \mathrm{m}$ (a), 2.18 $\mu \mathrm{m}$ (b) and $5.23 \mu \mathrm{m}$ (c). 
In the following we adopt this analytical expression to fit the experimental data and refine the size of the microsphere (which was roughly measured by conventional microscopy). However, before fitting experimental results with theoretical ones, we need to establish the mode number $n$ as well. By using the simplified equation $\pi \mathrm{md} \cong \mathrm{n} \lambda$ for WGMs in a spherical cavity, we estimate $n=46$ at $\lambda=600.7 \mathrm{~nm}, \mathrm{n}=19$ at $\lambda=622.8 \mathrm{~nm}$ and $\mathrm{n}=43$ at $\lambda=640.9 \mathrm{~nm}$ in spectra shown in Fig. 5,a Fig.5,b and Fig. 5,c, respectively. By substituting the assumed size of the sphere and mode number into Eq. 5, the calculated data of mode spacing $\left(\lambda_{n}-\lambda_{n+1}\right)$ vs the emission wavelength of the WGM peaks can be obtained. The sum of square difference of mode spacing between experimental and theoretical data was used as a criterion of fitting.

The calculated and experimentally observed $\Delta \lambda$ values for modes, which differ by one unit of angular momentum, are shown in Fig.6, demonstrating good agreement and lending support to the validity of the adopted method. By this way the size of microsphere is determined to be $5.11 \mu \mathrm{m}, 2.18 \mu \mathrm{m}$ and $5.23 \mu \mathrm{m}$ for data shown in Fig.6,a, Fig. 6,b and Fig. 6,c, respectively.

\section{CONCLUSION}

We have experimentally studied the three-dimensional imaging of the microspheres by using the confocal and conventional scanning polarization microscope. Our result show that the quality of the spherical microcavity, the spatial emission distribution and defect locations can be controlled combining emission confocal imaging technique with three-dimensional bright-field analysis. Also we have demonstrated the resonance modes in a novel composite system consisting of a spherical microcavity and thin CdTe NCs shell, which should be useful for a variety of photonic applications. Based on mode spacing treatments, we have developed a method to determine the size of the microsphere.

\section{REFERENCES}

1. $\quad$ K.J. Vahala, "Optical microcavities", Nature, 424, 839-846, 2003.

2. R.K. Chang, A.J. Campillo, Optical Processes in Microcavities, World Scientific, Singapore, 1996.

3. W. Inami, Y. Kawata, "Three-dimensional imaging analysis of confocal and conventional polarization microscopes by use of Mie scattering theory", Appl. Opt., 39, 6369-6373, 2000.

4. A. Serpenguzel, S. Arnold, G. Griffel, J.A. Lock, "Enhanced coupling to microsphere resonances with optical fibers", J. Opt. Soc. Am. B, 14,790-795, 1997.

5. M. Fayngold, "Spherical particle imaging and glare-ring spectroscopy with backscattered light", J. Opt. Soc. Am. A, 14, 1825-1835, 1997.

6. M.V. Artemyev, U. Woggon, "Quantum dots in photonic dots", Appl. Phys. Lett., 76,1353-1355, 2000.

7. X.D. Fan, M.C. Lonergan, Y.Z. Zhang, H.L. Wang, "Enhanced spontaneous emission from semiconductor nanocrystals embedded in whispering gallery optical microcavities", Phys. Rev. B, 64, 115310-1/4, 2001.

8. Y.P. Rakovich, L. Yang, E.M. McCabe, J.F. Donegan, T. Perova, A. Moore, N. Gaponik, A. Rogach, "Whispering Gallery Mode Emission from a Composite System of CdTe Nanocrystals and a Spherical Microcavity", Sem. Sci. Techn., 18, 914-918, 2003.

9. A.S. Susha, F. Caruso, A.L. Rogach, G.B. Sukhorukov, A. Kornowski, H. Möhwald, M. Giersig, A. Eychmüller, H. Weller, "Formation of luminescent spherical core-shell particles by the consecutive adsorption of polyelectrolyte and $\mathrm{CdTe}(\mathrm{S})$ nanocrystals on latex colloids", Coll. and Surf. A, 163, 3944, 2000.

10. N. Gaponik, D.V. Talapin, A.L. Rogach, K. Hoppe, E.V. Shevchenko, A. Kornowski, A. Eychmüller, H. Weller, "Thiol-capping of CdTe nanocrystals: an alternative to organometallic synthetic routes". J. Phys. Chem. B, 106, 7177-7185, 2002.

11. T. Wilson, R. Juškaitis, "On the extinction coefficient in confocal polarization microscopy". J. Microscopy, 179, 238-240, 1995.

12. P. Chýlek, "Resonance structure of Mie scattering: distance between resonances", J. Opt. Soc. Am. A, 7, 1609-1613, 1990. 